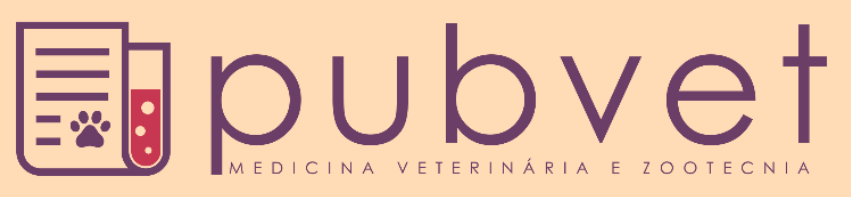

ISSN $1982-1263$

https://doi.org/10.31533/pubvet.v13n5a326.1-9

\title{
Osteopatia hipertrófica em cadela secundária a metástase pulmonar de neoplasia mamária: Relato de caso
}

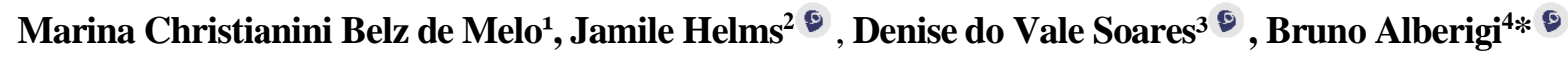 \\ ${ }^{1}$ Discente do curso de Medicina Veterinária da Universidade Castelo Branco, Rio de Janeiro - RJ Brasil. \\ ${ }^{2}$ Médica Veterinária, Centro veterinário Vida de cão, Rio de Janeiro - RJ, Brasil. \\ ${ }^{3}$ Médica Veterinária, Centro veterinário Vet Vargem, Rio de Janeiro - RJ, Brasil. \\ ${ }^{4}$ Professor do curso de Medicina veterinária da Universidade Castelo Branco, Rio de Janeiro - RJ, Brasil. \\ *Autor para correspondência, E-mail: bruno.alberigi@castelobranco.br
}

\begin{abstract}
Resumo. A osteopatia hipertrófica é uma desordem paraneoplásica caracterizada por neoformação óssea periosteal que geralmente está associada a neoplasias primárias ou metástases pulmonares. O diagnóstico é realizado por meio do histórico, anamnese e exames físico e radiográfico. $\mathrm{O}$ tratamento baseia-se na regressão dos sinais clínicos com a remoção da causa primária, com o prognóstico variando de reservado a desfavorável de acordo com a possibilidade de resolução do processo primário. Foi atendido um paciente da espécie canina, fêmea, de doze anos de idade, sem raça definida, apresentando dor e aumento de volume em membros torácicos, claudicação e andar relutante. Com os achados do exame físico e exame radiográfico associado ao histórico de neoplasia mamária anterior, suspeitou-se de síndrome paraneoplásica óssea. O presente relato tem como objetivo descrever um caso de osteopatia hipertrófica secundária à metástase pulmonar de neoplasia mamária em uma cadela, descrevendo os achados clínicos e o exame radiográfico como apoio diagnóstico e sua evolução clínica.
\end{abstract}

Palavras chave: Cão, proliferação periosteal, síndrome paraneoplásica

\section{Hypertrophic osteopathy in dog secondary to pulmonary metastasis of breast neoplasia: Case report}

\begin{abstract}
Hypertrophic osteopathy is a paraneoplastic disorder characterized by periosteal bone neoformation that is usually associated with primary neoplasms or pulmonary metastases. The diagnosis is made through history, anamnesis, physical and radiographic examinations. The treatment is based on regression of clinical signs with removal of the primary cause, with the prognosis ranging from reserved to unfavorable according to the possibility of resolution of the primary process. A patient of the canine, female, 12-yearold female, without a defined breed, was treated with pain and enlargement in the thoracic limbs, lameness and reluctance to walk. With the findings of the physical examination and radiographic examination associated with the history of previous mammary neoplasia, bone paraneoplastic syndrome was suspected. The present report aims to describe a case of hypertrophic osteopathy secondary to pulmonary metastasis of breast neoplasia in a female dog, describing clinical findings and radiographic examination as diagnostic support and clinical evolution.
\end{abstract}

Keywords: Dog, periosteal proliferation, paraneoplastic syndrome 


\title{
Osteopatía hipertrófica en perra secundaria a metástasis pulmonar de neoplasia mamaria: Relato de caso
}

\begin{abstract}
Resumen. La osteopatía hipertrófica es un desorden paraneoplásico caracterizado por neoformación ósea periosteal que generalmente está asociada a neoplasias primarias o metástasis pulmonares. El diagnóstico se realiza por medio del histórico, anamnesis y exámenes físico y radiográfico. El tratamiento se basa en la regresión de los signos clínicos con la retirada de la causa primaria, con el pronóstico que varía de reservado a desfavorable de acuerdo con la posibilidad de resolución del proceso primario. Se atendió a un paciente de la especie canina, hembra, de doce años, sin raza definida, presentando dolor y aumento de volumen en miembros torácicos, claudicación y andar reticente. Con los hallazgos del examen físico y examen radiográfico asociado al historial de neoplasia mamaria anterior, se sospechó de síndrome paraneoplásico óseo. El presente relato tiene como objetivo describir un caso de osteopatía hipertrófica secundaria a la metástasis pulmonar de neoplasia mamaria en una perra, describiendo los hallazgos clínicos y el examen radiográfico como apoyo diagnóstico y su evolución clínica.
\end{abstract}

Palabras clave: Perro, proliferación periosteal, síndrome paraneoplásico

\section{Introdução}

A osteopatia hipertrófica $(\mathrm{OH})$ é uma desordem paraneoplásica caracterizada por neoformação óssea periosteal geralmente associada a neoplasias primárias ou metástases pulmonares (Withers et al., 2015), sendo descrita com maior frequência em humanos e cães (Hoffmann et al., 2017; Salgado \& Villegas, 2015; Withers et al., 2015), e raramente descrita em gatos (Ettinger \& Feldman, 2004; Salgado \& Villegas, 2015). A média de idade dos animais acometidos é de nove anos de idade e qualquer raça pode ser acometida (Fossum, 2014). Contudo, as raças de grande porte são as mais relatadas (Withers et al., 2015).

Sua fisiopatogenia não está completamente elucidada, porém é relacionada com aumento do fluxo sanguíneo nas extremidades (Ettinger \& Feldman, 2004; Hoffmann et al., 2017). Uma hipótese é que as neoplasias liberam substâncias vasoativas na circulação que favorecem anastomoses arteriovenosas locais, resultando em sangue mal oxigenado no local e comprometendo a microcirculação, causando hipóxia tecidual e proliferação periosteal de osso neoformado (Bernardi et al., 2013; Salgado \& Villegas, 2015; Withers et al., 2015). Outra hipótese está relacionada com a presença de lesões pulmonares, que cursem com interrupção da circulação pulmonar, seja por má formação congênita ou presença de nódulos pulmonares (primários ou metastáticos), ocorre a liberação de megacariócitos que podem atingir a circulação sistêmica e nos capilares são ativados e secretam fator de crescimento derivado de plaquetas e fator de crescimento endotelial (VEGF), que induzem o crescimento mesenquimal local. Além disso, o VEGF estimula a angiogênese, que tem papel fundamental na osteogênese (Callemeyn et al., 2016; Silveira et al., 2000).

Os sinais clínicos incluem aumento de volume das extremidades dos membros acometidos, podendo estar associado à dor no local, claudicação e andar relutante. Além disso, outras manifestações clínicas inespecíficas podem estar presentes, como febre, letargia, anorexia (Bernardi et al., 2013; Ettinger \& Feldman, 2004), secreção ocular, congestão episcleral (Ferreira et al., 2018; Hermeto et al., 2013; Withers et al., 2015) e sinais respiratórios como tosse, taquipneia ou dispneia (Bernardi et al., 2013; Ettinger \& Feldman, 2004).

O diagnóstico é realizado por meio de exame físico e exame radiográfico (Bernardi et al., 2013). O tratamento baseia-se na regressão dos sinais clínicos com a remoção da causa primária (Ettinger \& Feldman, 2004; Fossum, 2014; Grillo et al., 2007), com o prognóstico variando de reservado a desfavorável de acordo com a possibilidade de resolução do processo primário (Fossum, 2014).

Objetiva-se com o presente trabalho, relatar um caso de osteopatia hipertrófica secundária à metástase pulmonar de neoplasia mamária em uma cadela, descrevendo os achados clínicos e o exame radiográfico como apoio diagnóstico. 


\section{Osteopatia hipertrófica em cão}

\section{Relato de caso}

Foi atendido em um centro veterinário, no Rio de Janeiro, um cão S.R.D, fêmea, com 12 anos de idade, pesando $30 \mathrm{~kg}$ e escore de condição corporal 5/9, apresentando nódulo de aproximadamente 15 $\mathrm{cm}$ em glândula mamária torácica direita. No exame clínico geral o animal apresentava temperatura retal $38^{\circ} \mathrm{C}$, batimentos cardíacos $120 \mathrm{bpm}$ e movimentos respiratórios $20 \mathrm{mrpm}$. O diagnóstico foi neoplasia mamária, sendo o tratamento cirúrgico recomendado para remoção de toda cadeia mamária direita.

Anteriormente ao procedimento cirúrgico, foi realizada coleta de sangue para realização do hemograma completo, o qual apresentou anemia normocítica normocrômica (Ht. 34,5\%; - ref.: 37 a 55; Hb. 11,1g/dL - ref.: 12 a 18; VGM 70fL - ref.: 60 a 77; CHGM 32,2\% - ref.: 31 a 36), linfopenia absoluta (linfócitos: 655 céls/ $\mu 1$ - ref.: 1000 a 4800) e monocitose (monócitos: 1572 céls/ $\mu 1$ - ref.: 150 a 1350), anisocitose e policromasia discretas como principais alterações. Na avaliação bioquímica, todos os valores se encontravam dentro do intervalo de referência para a espécie.

Também foi realizada radiografia torácica em três projeções (Figura 1) para avaliar a presença de metástase pulmonar, que revelou discreto aumento de densidade da árvore brônquica, acompanhado de discreta marcação bronquial e intersticial de distribuição difusa, além de mineralização das paredes bronquiais, sugerindo uma bronquite discreta associada a processo senil.

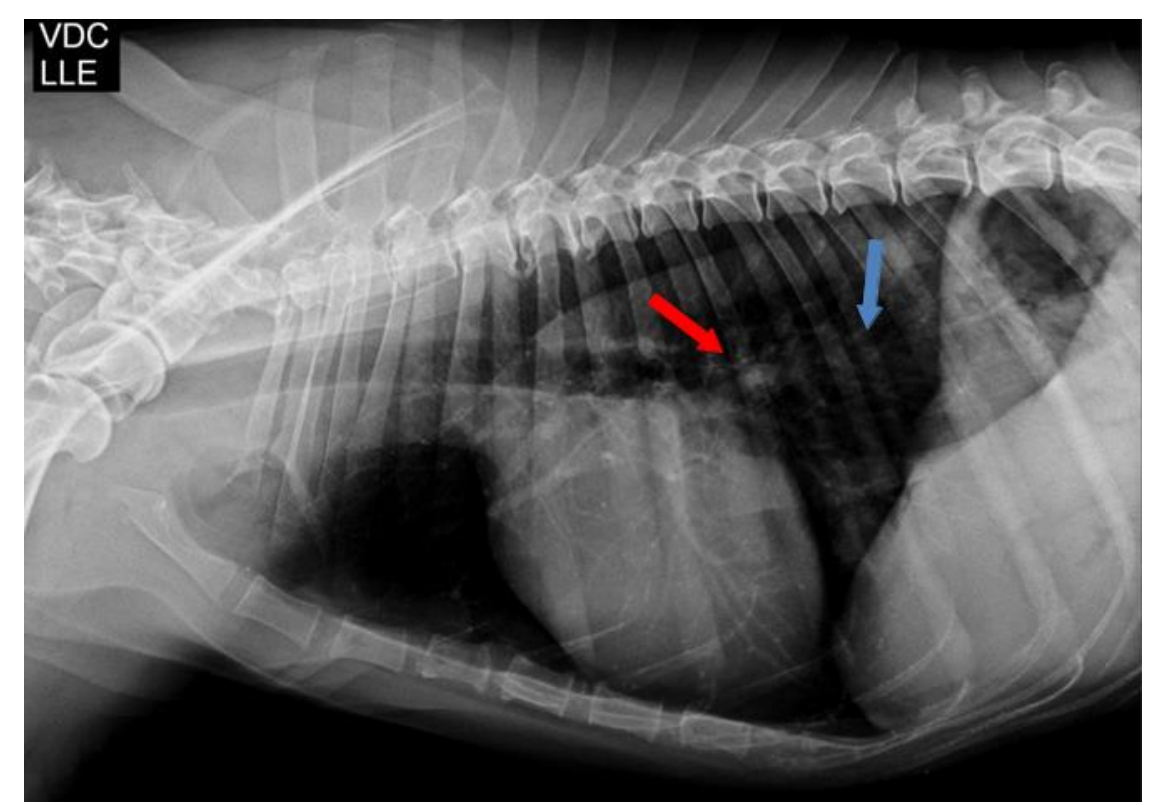

Figura 1. Radiografia simples de tórax, na projeção lateral esquerda, do paciente relatado, evidenciando discreto aumento de densidade da árvore brônquica e discreta marcação bronquial e intersticial de distribuição difusa (seta azul), além de mineralização das paredes bronquiais (seta vermelha), imagem sugestiva de bronquite discreta, associada a processo senil. Fonte: Luciana Sampaio.

Além destes, foi realizada ultrassonografia abdominal, que não visualizou sinais de metástases em cavidade abdominal. $\mathrm{O}$ tratamento instituído foi cirúrgico seguindo a técnica de mastectomia total unilateral direita com remoção dos linfonodos regionais (axilar e inguinal). $\mathrm{O}$ animal foi mantido internado no pós-cirúrgico imediato tendo alta médica no dia seguinte, sendo prescrito uso de cloridrato de tramadol quatro $\mathrm{mg} / \mathrm{kg}$ TID durante cinco dias, cefalexina $30 \mathrm{mg} / \mathrm{kg}$ BID durante 14 dias, dipirona $25 \mathrm{mg} / \mathrm{kg}$ BID, durante cinco dias, além de indicado alimentação com ração hipercalórica e suplementação com ômega 3.

O tecido orgânico removido cirurgicamente não foi submetido à avaliação histopatológica, pois o tutor não autorizou o envio do material. Animal retornou 10 dias após a cirurgia para retirada de pontos.

Sete meses após a cirurgia, o animal retorna à clínica com histórico de claudicação, fraqueza ao apoiar os membros no solo, aumento de volume distal em membros torácicos e apresentando sensibilidade à palpação do local ao exame físico, além de perda de peso evidente (peso $24,7 \mathrm{~kg}$ e escore de condição corporal 3/9). 
Foi prescrito meloxicam SID $(0,1 \mathrm{mg} / \mathrm{kg})$, durante sete dias e solicitado nova avaliação hematológica e radiografia dos membros acometidos (Figura 2). Além da anemia normocítica normocrômica (Ht. 32\%; VGM 70 fL; CHGM 33\%) relatada anteriormente, não foram encontradas outras alterações significativas no exame de sangue, contudo no exame radiográfico percebeu-se reação periosteal de aspecto irregular em toda a superfície óssea do rádio e da ulna, bilateralmente, sem descontinuidade da cortical óssea nestas regiões. Esta alteração radiográfica foi visualizada também no $1^{\circ}, 2^{\circ}$ e $5^{\circ}$ ossos dos metacarpos, bilateralmente.

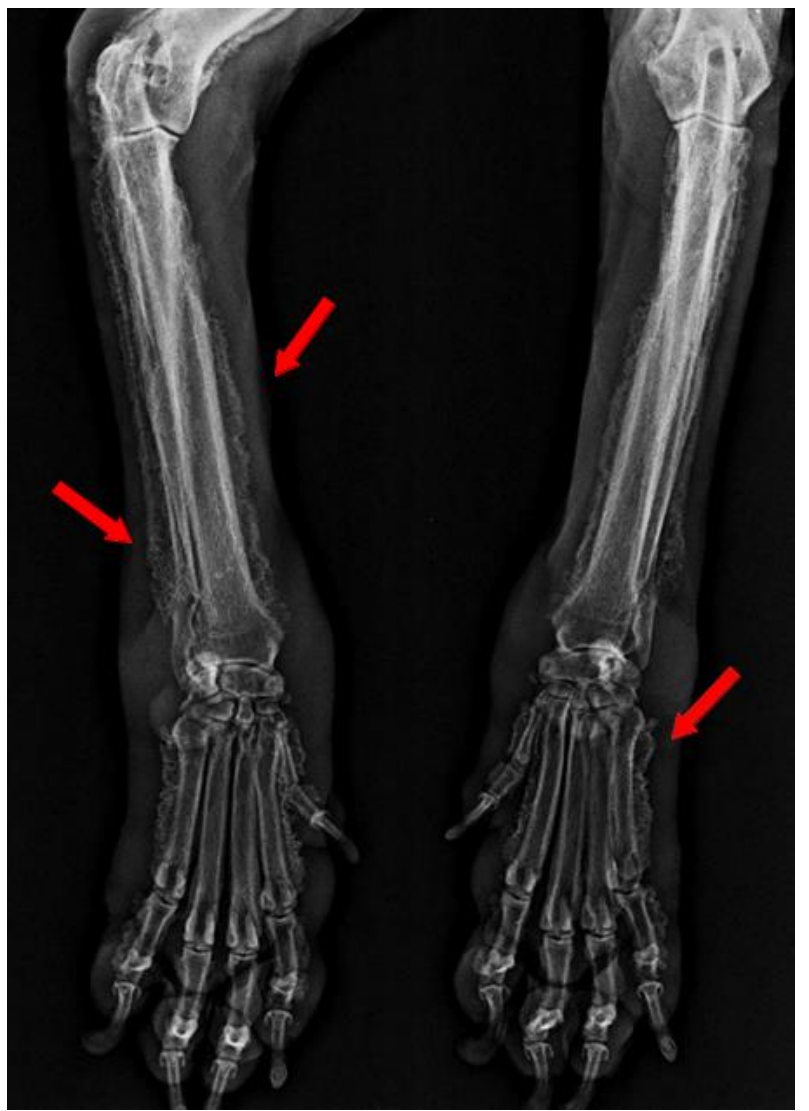

Figura 2. Radiografia de membros torácicos, na projeção dorsopalmar, evidenciando reação periosteal de aspecto irregular (setas vermelhas) em toda a superfície óssea do rádio e da ulna, bilateralmente, sem descontinuidade da cortical óssea nestas regiões. Fonte: Luciana Sampaio.

No retorno após uma semana, tutor relata melhora do animal ao apoiar os membros no solo após medicação, porém agora este apresentava tremores nos membros, dispneia discreta, hiporexia, sem episódios eméticos ou diarreia. Solicitado exame ultrassonográfico abdominal e radiográfico de tórax para pesquisa de metástase. Na ultrassonografia abdominal, não foi visualizado alterações dignas de nota, porém a radiografia torácica evidenciou além do discreto aumento de densidade da árvore brônquica já evidenciada no exame pré-operatório, duas formações ovaladas e de média radiodensidade, localizadas em lobo caudal esquerdo, sugerindo formações neoplásicas como possibilidade diagnóstica (Figuras 3 e 4 ).

Diante do quadro, a indicação foi apenas realizar o tratamento paliativo para melhorar a qualidade de vida do animal. Para o quadro álgico foi instituída a terapêutica com dipirona ( $25 \mathrm{mg} / \mathrm{kg}$ ) TID por 10 dias e prednisolona $(0,5 \mathrm{mg} / \mathrm{kg})$ BID por três dias e depois SID por 10 dias. Instruído retorno com o animal caso este apresente qualquer sintomatologia clínica, e caso permaneça estável retornar em 30 dias para revisão. Após 30 dias, o tutor não compareceu a clínica com o animal para o retorno préagendado, sendo assim, a médica veterinária deixou o tempo passar e após quatro meses entrou em contato com o tutor para obter notícias do paciente, sendo informada de que o mesmo veio a óbito poucos dias antes. 


\section{Osteopatia hipertrófica em cão}

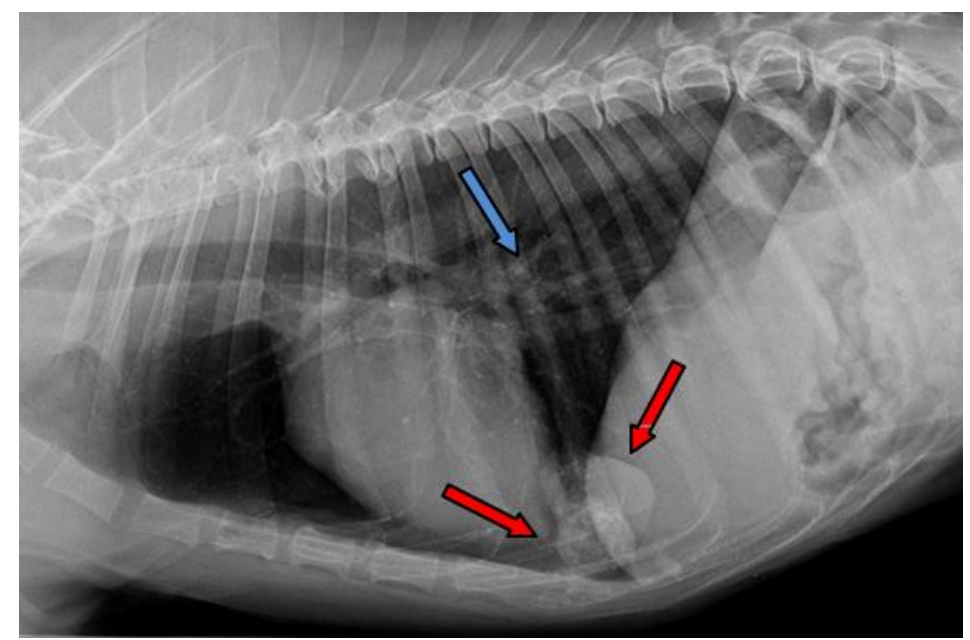

Figura 3. Radiografia simples de tórax, na projeção lateral direita, do paciente relatado, evidenciando padrão bronquial (seta azul) e formações ovaladas e de média radiodensidade localizadas em lobo caudal esquerdo (seta vermelha), sugestivo de metástase. Fonte: Luciana Sampaio

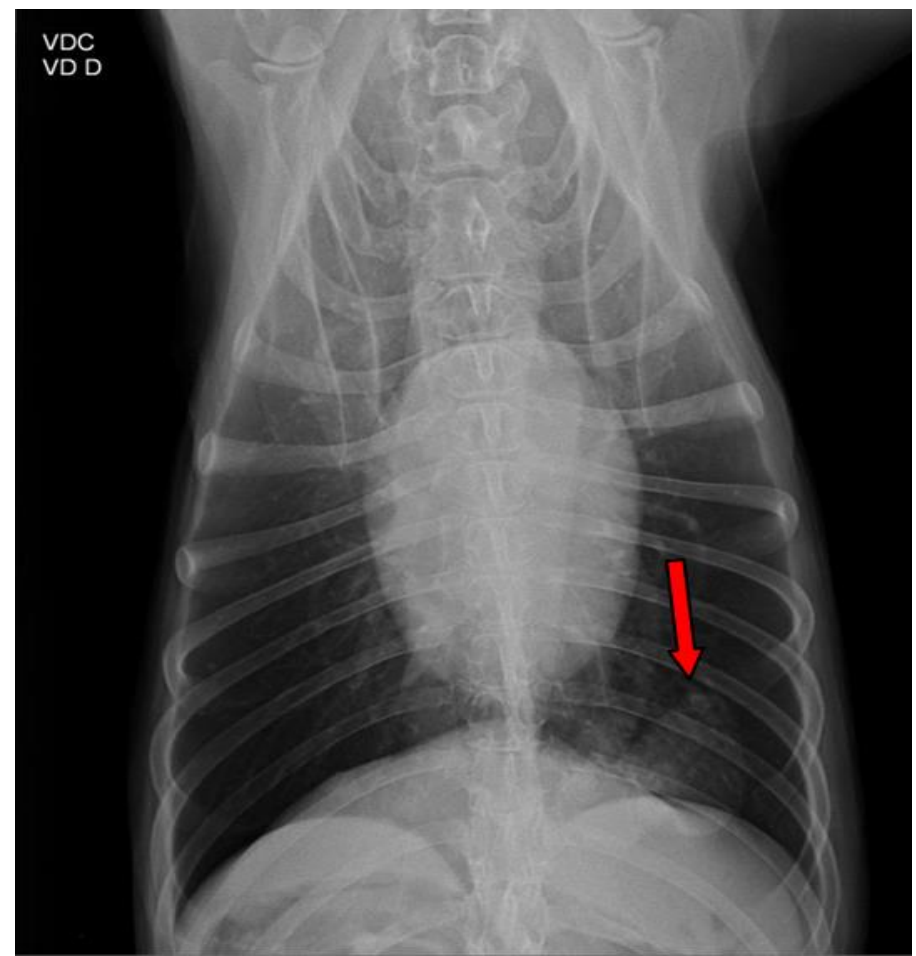

Figura 4. Radiografia simples de tórax, na projeção ventro-dorsal, do paciente relatado, evidenciando formação ovalada e de média radiodensidade localizada em lobo caudal esquerdo (seta vermelha), sugestivo de metástase pulmonar. Fonte: Luciana Sampaio

Associando os achados do exame físico com o histórico de neoplasia mamária anterior e presença de neoformação intratorácica, se estabeleceu a suspeita clínica de osteopatia hipertrófica como síndrome paraneoplásica (Ferreira et al., 2018; Withers et al., 2015).

\section{Discussão}

O presente relato retrata a ocorrência de uma síndrome paraneoplásica caracterizada por neoproliferação óssea periosteal, que pode acometer qualquer raça (Fossum, 2014), contudo Withers et al. (2015) descrevem um maior acometimento nas raças de grande porte. Neste caso, o animal relatado não possui raça definida, porém em virtude de seu peso e tamanho, é facilmente classificado como um cão de grande porte, sendo assim, condizente com a predisposição descrita na literatura. 
A presença de claudicação, fraqueza ao apoiar os membros ao solo, aumento de volume distal em membros torácicos e sensibilidade à palpação local, é considerado um achado frequente em cães portadores de OH (Bernardi et al., 2013; Ettinger \& Feldman, 2004), uma vez que o remodelamento ósseo cursa com processo inflamatório que culmina no edema de membros e a dor devido à ação de prostaglandinas, traduzida como andar dificultoso, manifestado clinicamente pela claudicação secundária ao estímulo álgico do ato de caminhar.

O animal apresentava emaciação e caquexia (escore de condição corporal 3/9) evidentes, provavelmente ocasionadas pela neoplasia mamária. Ferreira e colaboradores (2018) descrevem a caquexia como uma síndrome paraneoplásica de manifestação gastrointestinal em que ocorre alteração no metabolismo e desvio de nutrientes para o foco tumoral, resultando em perda de peso crônica, como observado no presente relato. O paciente apresentava hiporexia relatada pelo tutor, acredita-se que esse processo tenha ocorrido em virtude da liberação de fatores anorexígenos, contribuindo para o estado de caquexia do mesmo.

O manejo nutricional de pacientes portadores de caquexia neoplásica deve se basear em dietas com alto teor de proteínas e gordura e baixo teor de carboidratos (Daleck et al., 2016; Ferreira et al., 2018), sendo utilizada neste caso uma ração comercial capaz de atender as exigências nutricionais do animal mesmo em pequenos volumes. Com o acentuado catabolismo proteico que ocorre em pacientes com câncer, é preciso fornecer um maior aporte de aminoácidos para atender a demanda. $\mathrm{O}$ fornecimento de gordura em maior quantidade auxilia na manutenção do equilíbrio energético do animal, uma vez que a gordura é um componente de grande digestibilidade e de maior teor energético da dieta. (Daleck et al., 2016; Ferreira et al., 2018). O ômega 3 é um ácido graxo poliinsaturado que compõe a membrana celular e atua na permeabilidade das células, na regulação hormonal, processos inflamatórios e imunológicos, além de atuar como fonte energética (Daleck et al., 2016; Ferreira et al., 2018), sendo a sua suplementação benéfica para o paciente do presente relato.

A imagem radiográfica da $\mathrm{OH}$ pode sofrer algumas variações, contudo a presença de linhas ou faixas radio-transparentes anormais no interior das metáfises distais de ossos longos e neoformação óssea periosteal com a progressão da doença são frequentemente vistas. As imagens do caso relatado são semelhantes às encontradas por Bernardi et al. (2013). Segundo Prata (2016), a tomografia computadorizada apresenta maior sensibilidade que a radiologia para detectar lesões osteolíticas e de osteogênese, porém somente a radiografia dos membros foi realizada neste caso, uma vez que foi sensível para detectar as alterações relacionadas à osteopatia hipertrófica.

No presente relato, foi realizada apenas radiografia digital torácica pré-cirúrgica, que no primeiro momento não determinou a presença de neoplasias ou infecções pulmonares. Sete meses após o tratamento cirúrgico, foi realizada nova avaliação radiográfica do tórax, que evidenciou presença de duas formações ovaladas e de média radio-densidade, localizadas em lobo caudal pulmonar esquerdo, sugerindo metástase pulmonar, corroborando com os estudos de Withers et al. (2015), que relacionou a osteopatia hipertrófica com neoplasias primárias ou metástases pulmonares. A tomografia computadorizada com contraste também é o exame mais sensível na detecção de lesões pulmonares, e poderia ter revelado micro metástases no primeiro momento (Fossum, 2014; Otoni et al., 2010); porém, devido custo do exame, na maioria das vezes a radiografia torácica precede este método de imagem devido sua maior acessibilidade e baixo custo, sendo um dos pilares do estadiamento tumoral em pacientes oncológicos (Prata, 2016). Além disso, a radiografia digital apresenta maior sensibilidade que as radiografias convencionais, permitindo a detecção de nódulos pulmonares de menor tamanho (Mattoon \& Bryan, 2013).

Assim como relatado, a anemia é um achado frequente em pacientes oncológicos, sendo também uma manifestação paraneoplásica comum em cães e gatos, podendo estar relacionada com inflamação da doença crônica, hemólise e perda por sequestro celular. Além disso, em pacientes não submetidos à quimioterapia, pode ocorrer disfunção no metabolismo do ferro e sequestro do mesmo por ação de citocinas como a IL-1 (interleucina 1) e TNF (fator de necrose tumoral) secretados pelo tumor, o que reduz a meia vida das hemácias e diminui a resposta da medula óssea (Withrow et al., 2014). 


\section{Osteopatia hipertrófica em cão}

Apesar de a literatura descrever a presença de leucocitose em pacientes oncológicos como achado mais frequente, possivelmente por estimulação do sistema imune e pela condição inflamatória em decorrência da neoplasia mamária (Silva et al., 2014), no presente relato, o hemograma do animal relatado evidenciou leucometria normal (13.100 céls/ $\mu 1$ - ref.: 6.000 a 17.000), pois provavelmente a inflamação não foi suficiente para ocasionar um deslocamento do pool de neutrófilos da periferia dos vasos para a circulação (Thrall, 2015). A linfopenia absoluta observada (linfócitos: 655 céls/ $\mu 1$ - ref.: 1000 a 4800), pode estar relacionada a uma diminuição da produção, destruição ou ainda utilização dos linfócitos pelo tumor. Sugere-se também que tumores mais agressivos acarretam diminuição no número de linfócitos T em decorrência da inibição dos mesmos pelas células T regulatórias (Garrido et al., 2015). Além disso, foi evidenciada monocitose (1527 cels/ $\mu 1$ - ref.:150 a 1350), que é comumente relacionada com um processo inflamatório crônico. Sugere-se que a cronicidade da neoplasia possa acarretar uma resposta inflamatória sistêmica, piorando o prognóstico (Silva et al., 2014).

A elevação dos níveis de fosfatase alcalina está entre as anormalidades bioquímicas mais comuns em pacientes oncológicos (Karayannopoulou et al., 2006). A fosfatase alcalina possui as isoenzimas hepática, óssea e induzida por corticosteroides (Karayannopoulou et al., 2006). A isoenzima óssea da fosfatase alcalina reflete a atividade osteoblástica, que se encontra aumentada na doença em decorrência da proliferação óssea. Todavia, de acordo com Bernardi et al. (2013), elevação dos níveis de FA pode estar associada a diversos processos neoplásicos, não sendo específico de osteopatia hipertrófica. Além disso, o aumento também já foi relacionado com tumores mamários em cães (Karayannopoulou et al., 2006). Apesar de descrito na literatura, os níveis de fosfatase alcalina não se encontravam elevados no presente relato. Possivelmente, a injúria óssea ainda não tenha sido suficiente para aumentar os valores da FA. Ainda, neste caso, a fração óssea não foi mensurada separadamente, não sendo possível afirmar que esta poderia ou não estar aumentada.

O presente relato trata de uma síndrome paraneoplásica subdiagnosticada, apesar do fácil diagnóstico, sendo o tratamento difícil, pois depende da possibilidade de resolução da enfermidade primária. Neste caso, como não era possível à resolução do fator primário, somente cuidados paliativos foram adotados como medida terapêutica. Alinhado com a literatura, foi instituído o tratamento sintomático com uso de prednisolona, por via oral, sendo esta a droga de eleição devido aos seus efeitos anti-inflamatórios (Ferreira et al., 2018). Outras opções terapêuticas poderiam ter sido adotadas como o uso dos bisfosfonatos, pois são capazes de inibir a reabsorção óssea pela inibição dos osteoclastos (Ferreira et al., 2018; Milner et al., 2004). Em humanos é conhecido que além da sua ação inibitória nos osteoclastos, que auxilia no controle da osteólise e dor óssea, é descrito na literatura que estes fármacos também são capazes de atuar diretamente sobre as células neoplásicas prejudicando o desenvolvimento do tumor (Milner et al., 2004). No caso relatado, optou-se por não utilizar esta classe de drogas por falta de estudos mais detalhados em relação a dosagem, efeitos tóxicos e eficácia na espécie canina.

Em decorrência da condição financeira do tutor, não foi realizado exame histopatológico para classificação histológica do tumor mamário, impossibilitando a instituição da quimioterapia mais adequada para a neoplasia em questão. Sendo assim, não é possível afirmar que a metástase pulmonar e as SPN poderiam ter sido evitadas caso a quimioterapia fosse associada ao tratamento cirúrgico de remoção do tumor (Alves et al., 2017; D’Assis et al., 2016).

Poderia ter sido realizada uma biópsia guiada por ultrassom do nódulo pulmonar na tentativa de classificar os tipos de células neoplásicas e assim direcionar para o tratamento quimioterápico paliativo, a fim de diminuir a sintomatologia da osteopatia hipertrófica e melhorar a qualidade de vida do animal (Barros \& Repetti, 2015; Rodigheri, 2013). Porém, levando em conta o prognóstico desfavorável do quadro e a sua condição financeira, o tutor optou por não realizar os procedimentos.

\section{Conclusão}

Com o presente relato é possível concluir que é de extrema importância a realização dos exames préoperatórios de estadiamento oncológico, assim como o acompanhamento destes pacientes, pois é possível diagnosticar complicações como as síndromes paraneoplásica e metástases, mesmo meses após a retirada cirúrgica do tumor primário. 


\section{Referências bibliográficas}

Alves, R. O. D., Silva, V. F., Rodrigues, L. A., Xavier, D. C. R., Silva, P. L. N. \& Murta, D. V. F. (2017). Caracterização clínica e histopatológica de tumores mamários em cadelas atendidas em Viçosa, MG. Journal of Health \& Biological Sciences, 6(1):17-22.

Barros, V. T. M. \& Repetti, C. S. F. (2015). Metronomic chemotherapy in dogs: a review. Revista Portuguesa de Ciência Veterinárias, 110593-594.

Bernardi, C. A., Sanches, O. C., Gomes, D. R. \& Yamasaki, L. (2013). Osteopatia hipertrófica associada à metástase intratorácica de carcinoma mamário: Relato de caso. Paper presented at the Colloquium Agrariae.

Callemeyn, J., Van Haecke, P., Peetermans, W. E. \& Blockmans, D. (2016). Clubbing and hypertrophic osteoarthropathy: insights in diagnosis, pathophysiology, and clinical significance. Acta Clinica Belgica, 71(3):123-130.

D’Assis, M. J. M. H., Silva, L. P., Machado, M. C. d. A., Nascimento, N. A., Damasceno, K. A., Cassali, G. D. \& Lima, A. E. (2016). Carcinoma lobular pleomórfico de mama em cadela submetida ao tratamento quimioterápico com carboplatina-Relato de caso. Revista Brasileira de Medicina e Veterinária, 38139-146.

Daleck, C. R., Fonseca, C. S. \& Canola, J. C. (2016). Oncologia em cães e gatos. Rio de Janeiro: Roca.

Ettinger, S. \& Feldman, E. (2004). Tratado de medicina interna veterinária: doenças do cão e do gato. Rio de Janeiro: Guanabara Koogan.

Ferreira, M. G. P. A., Rodigheri, S. M., Reis Filho, N. P., Pascoli, A. L., Pazzini, J. M., Huppes, R. R., . . Nardi, A. B. (2018). Síndromes paraneoplásicas em cães e gatos: Parte 1. Revista Cólegio Brasileiro de Cirúrgia e Anestesiologia Veterinária, 17(1):24-34.

Fossum, T. W. (2014). Cirurgia de pequenos animais (4 ed. Vol. 1). São Paulo: Elsevier Brasil.

Garrido, E., Castanheira, T. L. L., Vasconcelos, R. O., Machado, R. Z. \& Alessi, A. C. (2015). Alterações hematológicas em cadelas acometidas por tumores mamários. PUBVET, 9287-347.

Grillo, T. P., Brandão, C. V. S., Mamprim, M. J., Jesus, C. M. N., Santos, T. C. \& Minto, B. W. (2007). Hypertrophic osteopathy associated with renal pelvis transitional cell carcinoma in a dog. The Canadian Veterinary Journal, 48(7):745-747.

Hermeto, L. C., Fernandes, S. S. P., de Affonseca Jardim, P. H., Mattei, D. R. \& Pelisari, T. (2013). Osteopatia Hipertrófica Pulmonar: Alterações clínicas e radiográficas em um paciente canino. Archives of Veterinary Science, 18(2):50-55.

Hoffmann, N. M., Luz, M. T., Vieira, L. V., Silveira, M. F., Corrêa, P. S. \& Oliveira, D. M. M. C. (2017). Osteopatia hipertrófica secundária a osteossarcoma condroblástico extraesquelético em um cão. Acta Scientiae Veterinariae, 451-4.

Karayannopoulou, M., Polizopoulou, Z. S., Koutinas, A. F., Fytianou, A., Roubies, N., Kaldrymidou, E., ... Koutinas, C. K. (2006). Serum alkaline phosphatase isoenzyme activities in canine malignant mammary neoplasms with and without osseous transformation. Veterinary Clinical Pathology, 35(3):287-290.

Mattoon, J. S. \& Bryan, J. N. (2013). The future of imaging in veterinary oncology: learning from human medicine. The Veterinary Journal, 197(3):541-552.

Milner, R. J., Farese, J., Henry, C. J., Selting, K., Fan, T. M. \& Lorimier, L. P. (2004). Bisphosphonates and cancer. Journal of Veterinary Internal Medicine, 18(5):597-604.

Otoni, C. C., Rahal, S. C., Vulcano, L. C., Ribeiro, S. M., Hette, K., Giordano, T., . . Amorim, R. L. (2010). Survey radiography and computerized tomography imaging of the thorax in female dogs with mammary tumors. Acta Veterinaria Scandinavica, 521-10.

Prata, I. B. S. (2016). Utilidade da tomografia computadorizada em oncologia: estudo clínico em cães e gatos. Master of Science, Universidade do Porto, Porto, Porto, Portugal.

Rodigheri, S. M. (2013). Quimioterapia metronômica em cães e gatos revisão de literatura. Clínica Veterinaria, 18(105):40-48. 


\section{Osteopatia hipertrófica em cão}

Salgado, R. A. C. \& Villegas, J. C. G. (2015). Osteopatía hipertrófica secundaria a metástasis pulmonar de carcinoma mamario. Spei Domus, 11(22):64 - 68.

Silva, A. H. C., Silva, D. M., Ribas, C. R., Dittrich, R. L., Dornbusch, P. T. \& Guérios, S. D. (2014). Alterações no hemograma de cadelas com neoplasia mamária. Ciência Animal Brasileira, 15(1):8792.

Silveira, L. H., Martinez-Lavin, M., Pineda, C., Fonseca, M. C., Navarro, C. \& Nava, A. (2000). Vascular endothelial growth factor and hypertrophic osteoarthropathy. Clinical and Experimental Rheumatology, 18(1):57-62.

Thrall, M. A. (2015). Hematologia e Bioquímica Clínica Veterinária. São Paulo, Brasil: Editora Roca.

Withers, S. S., Johnson, E. G., Culp, W. T. N., Rodriguez Junior, C. O., Skorupski, K. A. \& Rebhun, R. B. (2015). Paraneoplastic hypertrophic osteopathy in $30 \mathrm{dogs}$. Veterinary and Comparative Oncology, 13(3):157-165.

Withrow, S. J., Page, R. \& Vail, D. M. (2014). Ssmall animal clinical oncology. St. Louis: Elsevier Health Sciences.

Recebido: 6 de abril, 2019.

Aprovado: 2 de maio, 2019.

Publicado: 21 de maio, 2019

Licenciamento: Este artigo é publicado na modalidade Acesso Aberto sob a licença Creative Commons Atribuição 4.0 (CC-BY 4.0), a qual permite uso irrestrito, distribuição, reprodução em qualquer meio, desde que o autor e a fonte sejam devidamente creditados. 